\title{
Detection of Different Levels of Resistance to Vancomycin among Staphylococcus aureus Isolated from Surgical Departments of Tanta University Hospitals
}

\author{
Maha Salah Elnady ${ }^{\text {* }}$, Marwa Mohamed Ezzat ${ }^{1}$, Azza Mahmoud Hassan", \\ Amal Abd El Tawab Hasheesh ${ }^{2}$ and Mina Samy Messiha ${ }^{1}$ \\ ${ }^{1}$ Department of Medical Microbiology and Immunology, \\ ${ }^{2}$ Department of Surgery, Faculty of Medicine, Tanta University, Egypt \\ *Corresponding author
}

\section{A B S T R A C T}

Keywords

S. aureus, MRSA, vancomycin resistance, E-test, VITEK 2, SA-RVS

Article Info

\section{Accepted:}

20 July 2021

Available Online:

10 August 2021
Antibiotic resistance is the most worrisome problem in treating bacterial infections. It is very important to maintain the clinical efficacy of vancomycin because of their critical role in preventing and treating life-threatening healthcare associated infections (HAIs). Therefore, this study aimed to determine the existence of different levels of vancomycin resistance among Staphylococcus aureus (S. aureus) strains in Surgical Departments of Tanta University Hospitals. This study was carried out on 200 patients who had evidence of infections. All specimens were cultured on blood and mannitol salt agarsand their antimicrobial susceptibility were investigated by modified Kirby-Bauer method and VITEK 2 system. Vancomycin susceptibility was assessed by E-test and vancomycin screen agar. $m e c A$, vanA and $v a n B$ genes were investigated by polymerase chain reaction (PCR). Methicillin resistance was detected in $70 \%$ of $S$. aureus isolates and mecAgene was detected in $68 \%$ of these isolates. The total percentage of $S$. aureus with reduced vancomycin susceptibility (SA-RVS) was $20 \%$. All isolates were negative for $v a n A$ and $v a n B$ genes. VITEK 2 system failed to detect any isolates of SA-RVS.

\section{Introduction}

Staphylococcus aureus is a major bacterial human pathogen causing a wide variety of clinical manifestations ranging from minor skin infection to life-threatening disease. Infections are both community-acquired and hospital-acquired (Piewngam et al., 2020). The mortality of $S$. aureus invasive infections has fallen from $80 \%$ in the pre-antibiotic era to $16 \%-30 \%$ over the past two decades. Further reductions in mortality below $20 \%$ have remained far-fetched despite the introduction of new antibiotics, rapid diagnostic and 
susceptibility testing, widespread antibiotic stewardship programs and improvements in therapeutic supportive care (Miller et al., 2020).

Treatment options remains challenging due to the emergence of multi-drug resistant (MDR) strains such as methicillin-resistant $S$. aureus (MRSA). In 2014 global report, the World Health Organization (WHO) listed MRSA as one of the seven pathogens of international concern and that has been associated with a high number of mortality and septic shock cases compared tomethicillin-sensitive $S$. aureus (MSSA) (Che Hamzah et al., 2019).

Poor infection control measures as well as continued indiscriminate exposure of humans and animals to antibiotics have resulted in this huge problem of acquisition and dissemination of MRSA. This in turn limits the choice of treatment for MRSA infections and leads to enormous increases in vancomycin use for therapeutic, prophylactic, and empirical strategies (Lakhundi et al., 2018).

Two main forms of $S$. aureus with reduced vancomycin susceptibility (SA-RVS) have been identified. One form is vancomycin intermediate $S$. aureus (VISA) strains, which have minimum inhibitory concentrations (MICs) to vancomycin of $4-8 \mu \mathrm{g} / \mathrm{ml}$. It is mainly result from changes in peptidoglycan biosynthesis and thickened cell walls. The second form of vancomycin resistance called vancomycin resistant $S$. aureus (VRSA)which have MICs to vancomycin of $\geq 16 \mu \mathrm{g} / \mathrm{ml}$ and usually resulted from the conjugal transfer of the vanA operon from a vancomycin-resistant Enterococcus faecalis (Lowy, 2003).

In 1997, the first VISA strain (Mu50) with MIC of $8 \mu \mathrm{g} / \mathrm{ml}$ was reported from Japan (Hiramatsu et al., 1997). In 2002, the first case of VRSA was reported in a diabetic patient in the USA (Goldrick, 2002). The VISA strains are generally believed to be initiated from heterogenous vancomycin intermediate $S$. aureus (hVISA), which is defined as an $S$. aureus strain with a vancomycin MIC within the susceptible range $(\leq 2 \mu \mathrm{g} / \mathrm{ml})$ by conventional methods, while a cell subpopulation is in the vancomycinintermediate range $(\geq 4 \mu \mathrm{g} / \mathrm{ml})$ (Alam et al., 2014). The VISA and its precursor hVISA were discovered almost 20 years ago and have continued to be a stumbling block in the chemotherapy of MRSA (Hiramatsu et al., 2014).

These isolates are difficult to identify by routine laboratory methods as the vancomycin MIC level is within the susceptible range. It is determined by a plating-based, population analysis profile-area under the curve (PAPAUC) method. Unfortunately, PAP-AUC method is time-consuming, labor-intensive, and costly which limits its use in routine clinical laboratories. A variety of alternative methods for detection of the heteroresistant phenotype have been evaluated with varying success(Satola et al.,2011).

The clinical isolates of SA-RVS have emerged within the past two decades becoming a serious public health concern. These infections lead to higher rates of vancomycin treatment failure and are associated with extended hospitalization, higher risk of persistent infection and elevated treatment costs (Shariati et al., 2020).

\section{Materials and Methods}

The present study was carried out in Medical Microbiology and Immunology Department, Faculty of Medicine and Central Research Laboratory, Tanta University, on 200 patients admitted to Surgical Departments in Tanta University Hospitals during the period of research from October 2018 to October 2020.The study included in patients admitted at these departments for more than 3 days and patients received antibiotics for more than 10 days without improvement. 
Written informed consents were obtained from all participants in this research. Ethical approval for this study was provided by Ethics and Research Committee, Faculty of Medicine, Tanta University.

\section{Collection of samples}

Specimens were collected under complete aseptic precautions. The samples included wound and burn swabs, sputum samples, endotracheal aspirates as well as urine samples. Specimens were labeled and delivered as soon as possible to the laboratory. When immediate delivery to the laboratory was not possible, the specimen was refrigerated at $4-6{ }^{\circ} \mathrm{C}$ (Cheesbrough, 2006)

All specimens were cultured on blood, nutrient and mannitol salt agars. The plates were incubated at $37^{\circ} \mathrm{C}$ for 24 hours and then the isolates in the primary plates were identified by colony morphology, Gram film and various biochemical reactions according to traditional microbiological methods such as catalase test, coagulase test.

These biochemical reactions were done from colonies on nutrient agar (Adesoji et al., 2019).

\section{Antibiotic susceptibility testing}

\section{Disc diffusion method}

All the isolates of $S$. aureus from blood agar were tested for antibiotic susceptibility by modified Kirby- Bauer disc diffusion method according to CLSI guidelines (CLSI, 2020).

The following antibiotics (Oxoid, UK) were used Penicillin $(10 \mu \mathrm{g})$, Clindamycin $(2 \mu \mathrm{g})$, Trimethoprim-sulfamethoxazole $(25 \mu \mathrm{g})$, Erythromycin $(15 \mu \mathrm{g})$, Tetracycline $(30 \mu \mathrm{g})$, Ciprofloxacin $(5 \mu \mathrm{g})$, Gentamicin $(10 \mu \mathrm{g})$, Cefoxitin $(30 \mu \mathrm{g})$ and Rifampin $(5 \mu \mathrm{g})$.

\section{Vancomycin E-test}

The MIC for vancomycin was detected by Etest strips according to the manufacturer's instructions(Liofilchem, Italy). Using sterile forceps, the E- test strips were applied to the inoculated agar surface with the MIC scale facing upwards, followed by incubation at $35^{\circ} \mathrm{C}$ for 24 hours. The MIC was read directly from the scale in terms of $\mu \mathrm{g} / \mathrm{ml}$ at the point where the edge of the inhibition ellipse intersects with the MIC test strip.

\section{Antibiotic susceptibility by VITEK 2 system}

The steps done according to the manufacturer's instructions(BioMérieux SA, France). The results were automatically analyzed by VITEK system and interpreted as sensitive, intermediate, or resistant according to the MIC of each tested drug.

\section{Vancomycin screen agar}

A stock solution of vancomycin was prepared by dissolving $500 \mathrm{mg}$ of vancomycin powder in $10 \mathrm{ml}$ of sterile distilled water (final concentration was $50 \mathrm{mg} / \mathrm{ml}$ ). Further dilution of 1:10 was done twice to produce a working solution of $0.5 \mathrm{mg} / \mathrm{ml}$ vancomycin. For the final preparation of vancomycin screening agar; six $\mathrm{ml}$ of $500 \mathrm{ml}$ prepared media were removed under complete aseptic precautions and replaced by $6 \mathrm{ml}$ of working solution of vancomycin to prepare vancomycin screening agar with $6 \mu \mathrm{g} / \mathrm{ml}$. A drop from $0.5 \mathrm{McF}$ arland $S$. aureus suspension was inoculated using a micropipette to spot a $10 \mu \mathrm{l}$ drop on the surface of vancomycin screening agars. Alternatively, a swab was dipped in the McFarland suspension, the excess liquid expressed, and used to inoculate the vancomycin agar screen plates. Plates were examined carefully at 24 and 48 hours with transmitted light. 
Presence of more than one colony of the strain or light film of growth is interpreted as reduced susceptibility to vancomycin. For quality control purposes, vancomycin-resistant Enterococcus faecalis ATCC 51299 and $S$. aureus ATCC 25923 were used as positive and negative controls, respectively (Othman et al., 2018).

\section{Conventional PCR}

\section{DNA extraction}

According to the manufacturer's instructions (Qiagen-Germany), the bacteria from culture plate were removed by the inoculation loop and suspended in $180 \mu \mathrm{l}$ of lysis buffer ATL by vigorous stirring. $20 \mu \mathrm{l}$ of proteinase $\mathrm{K}$ were added, mixed by vortexing and incubated at $56{ }^{\circ} \mathrm{C}$ for 30 minutes. The samples were centrifuged to remove drops from inside the lid (8000 rpm for 1 minute). $200 \mu \mathrm{l}$ of lysis buffer AL were added to the sample, mixed by pulse vortexing for 15 seconds, and incubated at $70{ }^{\circ} \mathrm{C}$ for 10 minutes. $200 \mu \mathrm{l}$ of ethanol (96$100 \%$ ) were added to the sample, mixed by pulse vortexing for 15 seconds, and incubated at $70{ }^{\circ} \mathrm{C}$ for 10 minutes.

The mixture from the previous step was carefully applied to the QIAamp Mini spin column (in a $2 \mathrm{ml}$ collection tube), the cap was closed and centrifuged at $8000 \mathrm{rpm}$ for 1 minute. $500 \mu \mathrm{l}$ of buffer AW1 were added to the sample and centrifuged at $8000 \mathrm{rpm}$ for 1 minute. $500 \mu 1$ of buffer AW2 were added and centrifuged at $14000 \mathrm{rpm}$ for 3 minutes.

The QIAamp Mini spin column was placed in a clean $1.5 \mathrm{ml}$ microcentrifuge tube, $200 \mu \mathrm{l}$ of buffer AE were added and centrifuged at 8000 rpm for 1 minute. The centrifuged elutes were taken for gene detection.

\section{Nucleic acid amplification}

According to the manufacture instructions
(Applied Biosystems-USA), primers in table 1 were prepared by concentration $0.4 \mu \mathrm{m}$. The reaction was performed at a defined volume of $25 \mu 1$ and included $10 \mu \mathrm{l}$ of sample, $12.5 \mu 1$ of TaqMan Universal Master Mix, $1 \mu \mathrm{l}$ of forward primer and $1 \mu \mathrm{l}$ of reverse primer. All components were mixed well and centrifuged. The tubes were placed in the thermal cycler to be amplified. For amplification of mecA gene, initial denaturation was at $94{ }^{\circ} \mathrm{C}$ for $4 \mathrm{~min}$, followed by 30 cycles of denaturation at $94{ }^{\circ} \mathrm{C}$ for $45 \mathrm{~s}$, annealing at $50{ }^{\circ} \mathrm{C}$ for $30 \mathrm{sec}$ and elongation at $72{ }^{\circ} \mathrm{C}$ for $1 \mathrm{~min}$, and a final extension at $72{ }^{\circ} \mathrm{C}$ for $3 \mathrm{~min}$. For amplification of vanA gene, initial denaturation occurred at $94{ }^{\circ} \mathrm{C}$ for $2 \mathrm{~min}$, followed by 30 cycles of denaturation at $94{ }^{\circ} \mathrm{C}$ for $1 \mathrm{~min}$, annealing at $54{ }^{\circ} \mathrm{C}$ for $1 \mathrm{~min}$, elongation at $72^{\circ} \mathrm{C}$ for $1 \mathrm{~min}$, and a final extension at $72^{\circ} \mathrm{C}$ for $10 \mathrm{~min}$. For the amplification of $v a n B$ gene, initial denaturation occurred at $94^{\circ} \mathrm{C}$ for $5 \mathrm{~min}$, followed by 30 cycles of denaturation at $94^{\circ} \mathrm{C}$ for $25 \mathrm{~s}$, annealing at $52^{\circ} \mathrm{C}$ for $40 \mathrm{~s}$, elongation at $72^{\circ} \mathrm{C}$ for $1 \mathrm{~min}$, and final extension at $72^{\circ} \mathrm{C}$ for 6 min (Bamigboye et al., 2018).

\section{Agarose gel electrophoresis of the amplified DNA}

The PCR products were visualized and photographed under UV light after electrophoresis for 45 minutes at $100 \mathrm{~V}$ through $1 \%$ agarose gel containing ethidium bromide $(1 \mu \mathrm{g} / \mathrm{ml})$.

\section{Statistical analysis}

Statistical presentation and analysis of the results of the present study was conducted and the data were presented as mean \pm standard deviation (SD) or number and percentage. Comparison between groups were calculated by ANOVA and chi-square tests using computer program Statistical Package for the Social Sciences (SPSS) V. 20. P- value less than 0.05 was considered significant. 


\section{Results and Discussion}

\section{Prevalence and distribution of bacterial isolates}

Out of the 200 samples, 155 (77.5\%) showed mono-microbial growth, 15 (7.5\%) had polymicrobial growth with 2 organisms while 30 $(15 \%)$ displayed no growth. Gram negative organisms were the most prevalent organisms isolated from studied patients (63.2\%), followed by Gram positive organisms (32.5\%). Of notice, S. aureus represented 27\% of total isolates.

\section{Antimicrobial susceptibility testing}

By disc diffusion method, all studied isolates (100\%) were resistant to penicillin. Of notice, 35 cases $(70 \%)$ from $S$. aureus isolated were cefoxitin-resistant. The lowest resistance was to rifampin (8\%) and clindamycin including inducible clindamycin resistance (ICR) (34\%). On the other hand, all cases $(100 \%)$ were sensitive to linezolid. By using VITEK 2 system, the studied isolates showed the highest resistance $(100 \%)$ to penicillin and fusidic acid. The lowest resistance was to rifampin (8\%) and clindamycin (36\%).

On the other hand, all cases (100\%) showed sensitivity to linezolid, tigecycline, teicoplanin and vancomycin. Table 2 shows comparison between resistance pattern of isolated $S$. aureus to different antimicrobial agents by disc diffusion method and VITEK 2 system. $70 \%$ of isolated S. aureus were resistant, while $30 \%$ of these isolates were sensitive to cefoxitin.

\section{Susceptibility pattern of isolated $S$. aureus to vancomycin by E-test}

In this study, $86 \%$ of $S$. aureus isolates were sensitive to vancomycin (MICs $\leq 2 \mu \mathrm{g} / \mathrm{ml}$ ), while $8 \%$ showed intermediate resistance to vancomycin (MICs 4-8 $\mu \mathrm{g} / \mathrm{ml}$ ) by E-test (figure 1). No VRSA strains were detected (MICs $8-16 \mu \mathrm{g} / \mathrm{ml}$ ). Of notice, $6 \%$ of $S$. aureus isolates had MIC value of $3 \mu \mathrm{g} / \mathrm{ml}$ (query hVISA).

\section{Growth on vancomycin screen agar}

In this study, $9(18 \%)$ of $S$. aureus isolates grew on vancomycin screen agar with more than one colony. Three of them had MIC $\leq 2$ $\mathrm{ug} / \mathrm{ml}$ (VSSA) by vancomycin E-test and 3 isolates had MIC of 4-8 ug/ml (VISA). The remaining 3 isolates had MIC of $3 \mathrm{ug} / \mathrm{ml}$, that were query hVISA. Of notice, 3 out of 4 VISA strains grew on vancomycin screen agar with $75 \%$ sensitivity in detection of VISA. $82 \%$ of isolates showed no growth on vancomycin screen agar.

Out of the 50 isolates of $S$. aureus, $10(20 \%)$ were SA-RVS; 4 isolates (8\%) were VISA and 6 isolates $(12 \%)$ were hVISA as diagnosed by E-test and vancomycin screen agar.

\section{Conventional PCR results}

Out of $50 \mathrm{~S}$. aureus isolates, $34(68 \%)$ were mecA positive, while $16(32 \%)$ were mecA negative (figure 2).van $A$ and van $B$ genes were not detected in any of $S$. aureus isolates.

Antimicrobial resistance is a growing problem in medicine, resulting in limitations in the antimicrobial choices available for treatment of severe infections and increasing the risk of morbidity and mortality in patients (Weissman et al., 2013).There is no need of a crystal ball to predict that vancomycin resistance is going to represent the most serious therapeutical challenge in $S$. aureus infections. It is more difficult to predict, on the other hand, how long linezolid and daptomycin will maintain their full activity against MRSA (Livermore, 2016).

In the present study, when the susceptibility of $S$. aureus was studied by disc diffusion 
method, it was found that the highest resistance rate was detected to penicillin (100\%), cefoxitin $(70 \%)$, gatifloxacin $(56 \%)$, erythromycin and gentamicin (54\%, each).

Also, S. aureus isolates showed resistance to ciprofloxacin and ofloxacin (46\%, each), tetracycline (44\%), doxycycline (42\%), trimethoprim-sulfamethoxazole $(40 \%)$ and clindamycin (34\%). However, these isolates exhibited highest sensitivity rates to linezolid $(100 \%)$ and rifampin (92\%).

Supportive to results of the current study, Kishket al., (2020) reported that erythromycin and clindamycin resistance by disc diffusion which detected in $S$. aureus isolates was $54.5 \%$ and $38.6 \%$, respectively. Additionally, Li et al., (2019) reported $100 \%$ sensitivity to linezolid in $S$. aureus isolates by disc diffusion method. Resistance rate to erythromycin, tetracycline and trimethoprimsulfamethoxazole was 53, 40 and $35 \%$, respectively.

One the other hand, the observed resistance for clinical $S$. aureus isolates in the study of Adesoji et al., (2019) demonstrated the highest resistance $(100 \%)$ to erythromycin and the lowest resistance $(27.5 \%)$ to ofloxacin and gentamycin (20\%).Also, higher resistance rate to fluoroquinolone class $(76.3 \%)$ was reported among tested $S$. aureus isolates in the study done by Kashef et al., (2020). The characteristics and antimicrobial resistance profiles of Staphylococci differs according to geographical regions and in relation to antibiotic usage. The prevalence of MRSA in the present study was $70 \%$ according to both cefoxitin disc diffusion and VITEK 2 MICs for oxacillin and cefoxitin. These results matched with the results obtained by AbdelMaksoud et al., (2016) from 12 hospitals in Egypt from 2005 to 2013, they reported that MRSA isolates constituted $76 \%$ of staphylococcal infections by disc diffusion method. Also, Amr and Gammal, (2017) in Zagazig University Hospitals detected the prevalence of MRSA by VITEK 2 system which was about $78 \%$ of $S$. aureus isolates.

On the other hand, the prevalence of MRSA in the present study was higher than that obtained in Egypt by Taha et al., (2019) reported lower prevalence rate of MRSA (20\%). Naimi et al., (2017) in Afghanistan, as they reported $56 \%$ of isolated $S$. aureus were MRSA by disc diffusion method. Also, Wangai et al.,(2019) reported between 2014 and 2016 the overall MRSA prevalence in East Africa was $53.4 \%$ by VITEK 2 system. In India, Raut et al., (2017) reported that the prevalence of MRSA was $43.6 \%$ by cefoxitin disc diffusion.

Regarding the prevalence of SA-RVS, Othman et al., (2018) revealed that out of their 100 S. aureus isolates, $22 \%$ were VISA. There were 9 isolates $(9 \%)$ grew on screening agar 6 $\mu \mathrm{g} / \mathrm{ml}$ and were designated as hVISA. All their $S$. aureus isolates were susceptible for both vancomycin and linezolid by VITEK 2 system. Abdel-Maksoud et al., (2016) reported that susceptibility to vancomycin was detected in $80.2 \%$ of MRSA isolates by E-test and VISA was detected in $1.2 \%$ of their isolates.

However, $18.6 \%$ of the isolates showed MIC $3 \mu \mathrm{g} / \mathrm{ml}$. VRSA was not detected among staphylococcal isolates. 
Table.1 The primers used and their product size ${ }^{(88)}$

\begin{tabular}{|c|c|c|}
\hline Primer name & Primer sequence $\left(5^{\prime}-3^{\prime}\right)$ & Product size (pb) \\
\hline mecAF & GTAGAAATGACTGAACGTCCGATAA & \multirow[t]{2}{*}{585} \\
\hline mecAR & CCAATTCCACATTGTTTCGGTCTAA & \\
\hline $\operatorname{van} A \mathbf{F}$ & GGCAAGTCAGGTGAAGATG & \multirow[t]{2}{*}{713} \\
\hline van $A \mathbf{R}$ & ATCAAGCGGTCAATCAGTTC & \\
\hline $\operatorname{van} B \mathrm{~F}$ & GTGACAAACCGGAGGCGAGGA & \multirow[t]{2}{*}{430} \\
\hline van $B \mathrm{R}$ & CCGCCATCCTCCTGCAAAAAA & \\
\hline
\end{tabular}

Fig.1 Vancomycin E-test showing intermediate isolate of $S$. aureus $(\mathrm{MIC}=6 \mu \mathrm{g} / \mathrm{ml})$

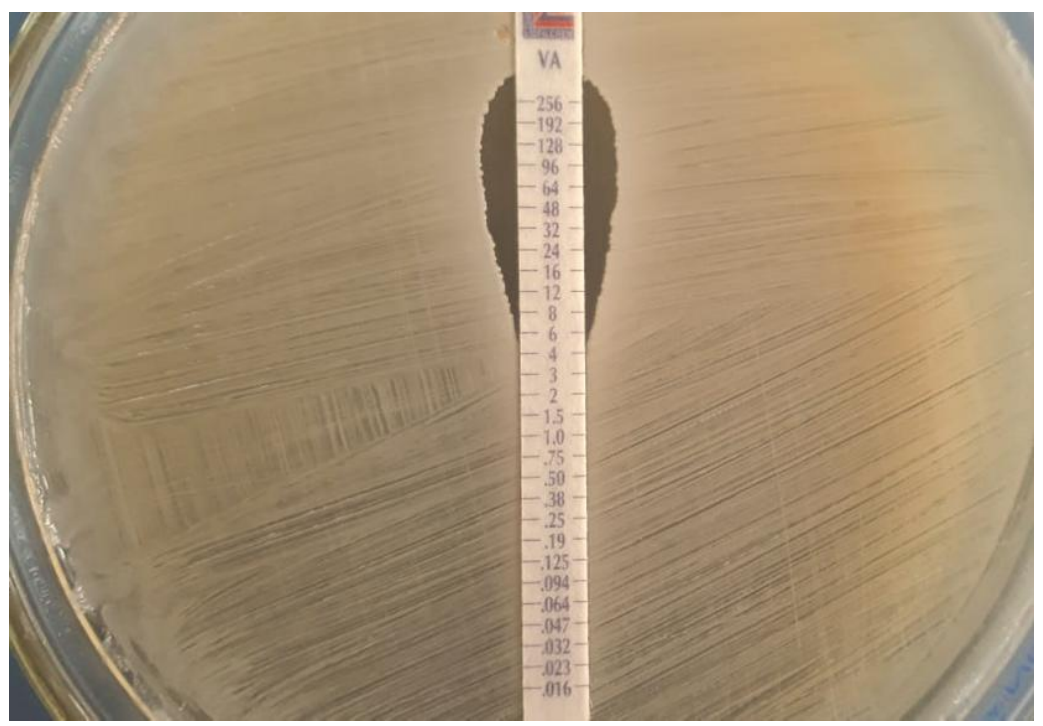

Fig.2 Gel electrophoresis of PCR amplification of mecAgene.

$\begin{array}{lllllllllll}1 & 2 & 3 & 4 & 5 & 6 & 7 & 8 & 9 & 10 & 11\end{array}$

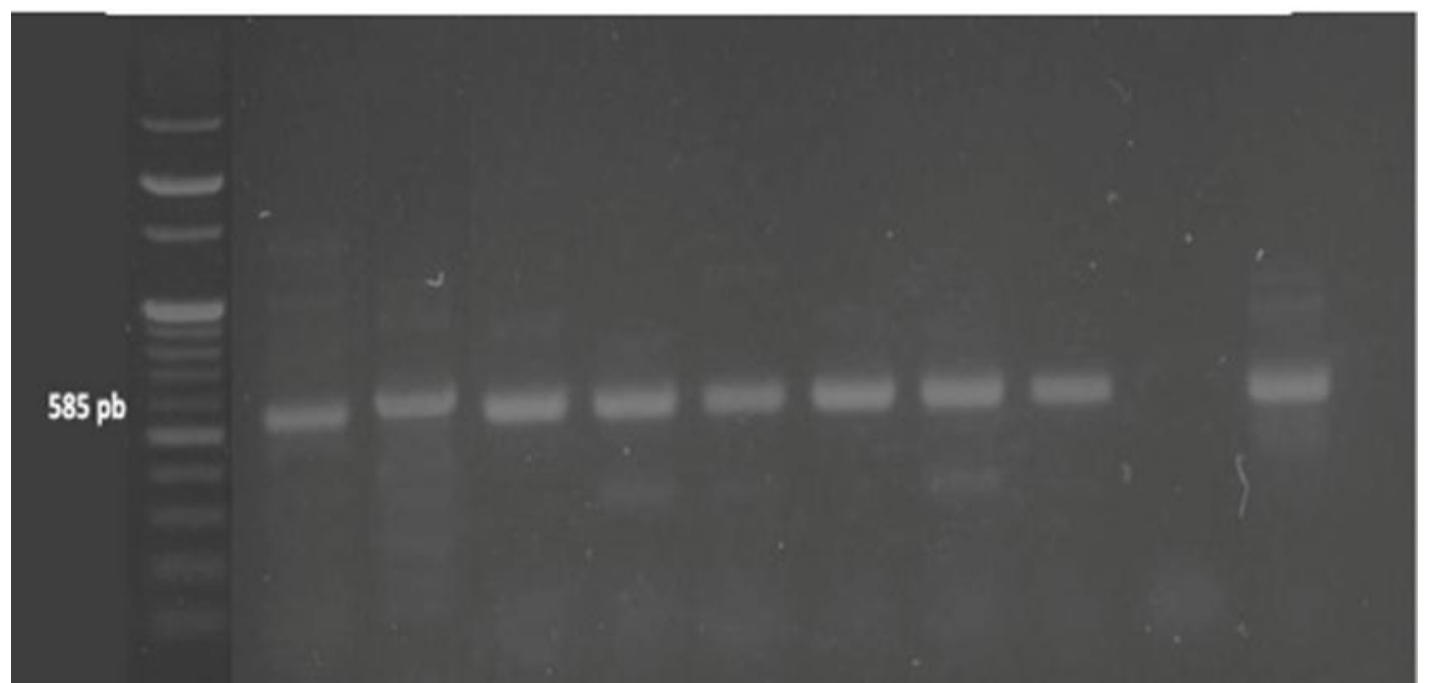

Lane $1=$ marker of $100 \mathrm{pb}$ DNA ladder; Lane $2=$ mecA positive control (585 pb) Lane $3,4,5,6,7,8,9$ and $11=m e c A$ positive isolates

Lane $10=m e c A$ negative isolate 
Table.2 Comparison between resistance pattern of isolated $S$. aureus to different antimicrobial agents by disc diffusion method and VITEK 2 system

\begin{tabular}{|c|c|c|c|c|c|c|c|c|c|c|c|c|}
\hline \multirow[t]{4}{*}{ Antibiotic } & \multicolumn{12}{|c|}{$\begin{array}{l}\text { S. aureusisolated } \\
\qquad(\mathrm{N}=50)\end{array}$} \\
\hline & \multicolumn{4}{|c|}{$\mathbf{S}$} & \multicolumn{4}{|c|}{ I } & \multicolumn{4}{|c|}{$\mathbf{R}$} \\
\hline & \multicolumn{2}{|c|}{$\begin{array}{c}\text { Disc } \\
\text { diffusion }\end{array}$} & \multicolumn{2}{|c|}{ VITEK 2} & \multicolumn{2}{|c|}{$\begin{array}{c}\text { Disc } \\
\text { diffusion }\end{array}$} & \multicolumn{2}{|c|}{ VITEK 2} & \multicolumn{2}{|c|}{$\begin{array}{c}\text { Disc } \\
\text { diffusion }\end{array}$} & \multicolumn{2}{|c|}{ VITEK 2} \\
\hline & No. & $\%$ & No. & $\%$ & No. & $\%$ & No. & $\%$ & No. & $\%$ & No. & $\%$ \\
\hline Penicillin & $\mathbf{0}$ & $\mathbf{0}$ & $\mathbf{0}$ & $\mathbf{0}$ & $\mathbf{0}$ & $\mathbf{0}$ & $\mathbf{0}$ & $\mathbf{0}$ & 50 & 100 & 50 & 100 \\
\hline Cefoxitin & 15 & 30 & 15 & 30 & $\mathbf{0}$ & $\mathbf{0}$ & $\mathbf{0}$ & $\mathbf{0}$ & 35 & 70 & 35 & 70 \\
\hline Erythromycin & 18 & 36 & 20 & 40 & 5 & 10 & 2 & 4 & 27 & 54 & 28 & 56 \\
\hline Gentamicin & 19 & 38 & 20 & 40 & 4 & 8 & 2 & 4 & 27 & 54 & 28 & 56 \\
\hline Ciprofloxacin & 25 & 50 & 25 & 50 & 2 & 4 & $\mathbf{0}$ & $\mathbf{0}$ & 23 & 46 & 25 & 50 \\
\hline Tetracycline & 25 & 50 & 27 & 54 & 3 & 6 & 1 & 2 & 22 & 44 & 22 & 44 \\
\hline $\begin{array}{l}\text { Trimethoprim- } \\
\text { sulfamethoxazole }\end{array}$ & 25 & 50 & 25 & 50 & 5 & 10 & 5 & 10 & 20 & 40 & 20 & 40 \\
\hline Clindamycin & 30 & 60 & 31 & 62 & 3 & 6 & 1 & 2 & 17 & 34 & 18 & 36 \\
\hline Rifampin & 46 & 92 & 46 & 92 & $\mathbf{0}$ & $\mathbf{0}$ & $\mathbf{0}$ & $\mathbf{0}$ & 4 & 8 & 4 & 8 \\
\hline Linezolid & 50 & 100 & 50 & 100 & $\mathbf{0}$ & $\mathbf{0}$ & $\mathbf{0}$ & 0 & $\mathbf{0}$ & 0 & 0 & $\mathbf{0}$ \\
\hline $\mathrm{X}^{2}$ & \multicolumn{4}{|c|}{0.153} & \multicolumn{4}{|c|}{2.571} & \multicolumn{4}{|c|}{0.093} \\
\hline P-value & \multicolumn{4}{|c|}{0.998} & \multicolumn{4}{|c|}{0.765} & \multicolumn{4}{|c|}{0.999} \\
\hline
\end{tabular}

S: susceptible. I: Intermediate. R: Resistant. $P \geq 0.05$ is not significant.

The different results between the 2 tests are highlighted.

In Iran, Asadpour and Ghazanfari(2019) found that of the 110 studied isolates, the vancomycin MIC was $>2 \mu \mathrm{g} / \mathrm{ml}$ in $10 \%$ isolates. Of these, three $(2.3 \%)$ VRSA and eight $(7.3 \%)$ VISA were identified. In India, Bhattacharya et al.,(2015) showed that out of $100 \mathrm{~S}$. aureus isolates, the prevalence of VRSA, VISA, and hVISA was found to be $3 \%, 12 \%$ and $6 \%$, respectively by E-test and $\mathrm{E}$ test glycopeptide resistance detection strips. Higher rates of vancomycin resistance were detected in a Nigerian study conducted by Olufunmiso et al., (2017)where15\% of the isolates were considered VISA and $44.5 \%$ of the isolates were considered VRSA.

A lower rate of VISA isolation among $S$. aureus (4\%) was reported in 2018byJahanshahi et al., Also, Baseri et al., (2018) revealed that the overall prevalence rate of VISA was $0.09 \%$ in Iran between
2010-2017. The relevance of hVISA and its accurate epidemiology will remain controversial until a reliable and practical testing method becomes standardized and adopted for use in clinical laboratories and future studies (Walsh et al., 2001).

Supportive to the results of the current study, Swenson et al., (2009) reported that the VITEK 2 system tended to categorize VISA isolates as susceptible.

This was justified by Edwards et al.,(2012) who demonstrated that MICs from automated systems and the E-test were significantly lower after cryopreservation, if compared with those from the E-test analysis at the time of isolation.

In the present study, conventional PCR detected mecA gene in $68 \%$ of $S$. aureus 
isolates. Out of 35 phenotypically diagnosed MRSA isolates, 34 isolates were positive for mecA gene $(97.1 \%)$. Similar results were reported by Bhattacharya et al.,(2015) found that out of 47 phenotypically MRSA isolates, 46 was mecA positive (97.9\%). Sahebnasagh et al., (2014)found among 126 isolates of $S$. aureus, 98 of isolates were determined MRSA using disc diffusion method. Only 87 (69\%) isolates harbored the mecA gene.

On the other hand, Anand et al., (2009) found that out of the $50 \mathrm{~S}$. aureus isolates, 32 were resistant with cefoxitin disc diffusion. For these 32 isolates mecA gene was positive. Also, Sadeghi and Mansouri(2014) reported that $56.8 \%$ of their isolates were identified as MRSA and all were characterized by the presence of mecA gene. The methicillin resistance with negative $m e c A$ gene could be due to the presence of other resistance mechanisms, such as large amounts of produced beta-lactamase or change in mecAgene due to the mutations. Otherwise, it would be due to the lack of optimal PCR conditions(Sahebnasagh et al., 2014).

Regarding detection of van $A$ and van $B$ genes by PCR in this study, none of $S$. aureus isolates could demonstrate the presence of vanA or $v a n B$ gene by PCR.

Similar results were obtained in 2018 by Bamigboye et al., $61(83.6 \%)$ of the $S$. aureus isolates were VSSA, 11 (15.1\%) were VISA, and $1(1.4 \%)$ was VRSA.

All the VISA and VRSA isolates were vanA and $v a n B$ gene negative. Kumar (2016) in India found that among the $47 \mathrm{~S}$. aureus isolates, 2 isolates were highly resistant to vancomycin. PCR amplification of both isolates indicated presence of mecAand vanAgenes. Also, Thati et al., (2011) reported among the clinical isolates of $S$. aureus, sixteen isolates were VISA and seven isolates were VRSA. PCR amplification for vanA among the seven VRSA showed that six contained vanA, whereas all the isolates expressed mecA.

\section{Acknowledgments}

I would like to profusely thank the staff of Microbiology laboratory, Faculty of Medicine, Tanta University for their cooperation in the collection of isolates used in this study.

\section{Conflict of interest}

There is no conflict of interest between the authors.

\section{References}

Abdel-Maksoud M, El-Shokry M, Ismail G, Hafez S, El-Kholy A, Attia E and Talaat M. Methicillin-resistant Staphylococcus aureus recovered from healthcare- and community-associated infections in Egypt. Int $J$ Bacteriol 2016; 2016:5751785.

Adesoji A T, Onuh J P, Bagu J and Itohan S A. Prevalence and antibiogram study of Staphylococcus aureus isolated from clinical and selected drinking water of Dutsin-Ma, Katsina state, Nigeria. Afr Health Sci 2019; 19:138592.

Alam M T, Petit R A 3rd, Crispell E K, Thornton T A, Conneely K N, Jiang Y, Satola S W and Read T D. Dissecting vancomycin-intermediate resistance in Staphylococcus aureus using genomewide association. Genome Biol Evol 2014; 6:1174-85.

Amr G E and Gammal S A. Emergence of vancomycin resistant Staphylococcus aureus isolated from patients in ICUs of Zagazig University Hospitals. Egypt J Med Microbiol 2017; 26:53-9.

Anand K B, Agrawal P, Kumar S and Kapila 
K. Comparison of cefoxitin disc diffusion test, oxacillin screen agar and PCR for mecA gene for detection of MRSA. Indian J Med Microbiol 2009; 27:27-9.

Asadpour L and Ghazanfari N. Detection of vancomycin nonsusceptible strains in clinical isolates of Staphylococcus aureus in northern Iran. Int Microbiol 2019; 22:411-7.

Bamigboye B T, Olowe O A and Taiwo SS. Phenotypic and molecular identification of vancomycin resistance in clinical Staphylococcus aureus isolates in Osogbo, Nigeria. Eur $J$ Microbiol Immunol (Bp) 2018; 8:2530.

Baseri N, Najar-Peerayeh S, Bagheri Amiri F. Prevalence of vancomycinintermediate Staphylococcus aureus among clinical isolates in Iran: A systematic review and meta-analysis. $J$ Glob Antimicrob Resist 2018; 15:17887.

Bhattacharya S, Bir R and Majumdar T. Evaluation of multidrug resistant Staphylococcus aureus and their association with biofilm production in a Tertiary Care Hospital, Tripura, Northeast India. J Clin Diagn Res 2015; 9:1-4.

Che Hamzah A M, Yeo C C, Puah S M, Chua $\mathrm{K} \mathrm{H}$ and Chew C H. Staphylococcus aureus infections in Malaysia: A review of antimicrobial resistance and characteristics of the clinical isolates, 1990-2017. Antibiotics (Basel) 2019; 8:128.

Cheesbrough M. District laboratory practice in tropical countries. $2^{\text {nd }}$ ed. Cambridge, UK:Cambridge University press;2006.p.65-107.

Edwards B, Milne K, Lawes T, Cook I, Robb A and Gould IM. Is vancomycin MIC "creep" method dependent? Analysis of methicillin-resistant Staphylococcus aureus susceptibility trends in blood isolates from North East Scotland from 2006 to 2010. J clin microbiol 2012; 50:318-25.

Goldrick B. First reported case of VRSA in the United States: an alarming development in microbial resistance. Am J Nurs 2002; 102:17.

Hiramatsu K, Aritaka N, Hanaki H, Kawasaki S, Hosoda Y, Hori S, Fukuchi Y and Kobayashi I. Dissemination in Japanese hospitals of strains of Staphylococcus aureus heterogeneously resistant to vancomycin. Lancet 1997; 350:16703.

Hiramatsu K, Kayayama Y, Matsuo M, Aiba Y, Saito M, HishinumaT and Iwamoto A. Vancomycin-intermediate resistance in Staphylococcus aureus. $J$ Glob Antimicrob Resist 2014; 2:213224.

Jahanshahi A, Zeighami $\mathrm{H}$ and Haghi $\mathrm{F}$. Molecular characterization of methicillin and vancomycin resistant Staphylococcus aureus strains isolated from hospitalized patients. Microb Drug Resist 2018; 24:1529-36.

Kashef M T, Saleh N M, Assar N H and Ramadan M A. The antimicrobial activity of ciprofloxacin-loaded niosomes against ciprofloxacinresistant and biofilm-forming Staphylococcus aureus. Infect Drug Resist 2020; 13:1619-29.

Kishk R M, Anani M M, Nemr N A, Soliman $\mathrm{N} \mathrm{M}$ and Fouad $\mathrm{M}$ M. Inducible clindamycin resistance in clinical isolates of Staphylococcus aureus in Suez Canal University Hospital, Ismailia, Egypt. J Infect Dev Ctries 2020; 14:1281-7.

Lakhundi S and Zhang K. Methicillin-resistant Staphylococcus aureus: molecular characterization, evolution, and epidemiology. Clin Microbiol Rev 
2018; 31:109-18.

Li X, Huang T, Xu K, Li C and Li Y. Molecular characteristics and virulence gene profiles of Staphylococcus aureus isolates in Hainan, China. BMC Infect Dis 2019; 19:873.

Livermore D M. Can beta-lactams be reengineered to beat MRSA? Clin Microbiol Infect 2006; 12:11-6.

Lowy F D. Antimicrobial resistance: the example of Staphylococcus aureus. J Clin Invest 2003; 111:1265-73.

Miller L S, Fowler V G, Shukla SK, Rose W E and Proctor R A. Development of a vaccine against Staphylococcus aureus invasive infections: evidence based on human immunity, genetics and bacterial evasion mechanisms. FEMS Microbiol Rev 2020; 44:123-53.

Naimi H M, Rasekh H, Noori A Z and Bahaduri MA. Determination of antimicrobial susceptibility patterns in Staphylococcus aureus strains recovered from patients at two main health facilities in Kabul, Afghanistan. BMC Infect Dis 2017; 17:737.

Olufunmiso O, Tolulope I and Roger C. Multidrug and vancomycin resistance among clinical isolates of Staphylococcus aureus from different teaching hospitals in Nigeria. Afr Health Sci 2017; 17:797-807.

Othman H B, Halim R M A, Gomaa F A M and Amer M Z. Vancomycin M I C distribution among methicillinresistant Staphylococcus aureus. is reduced vancomycin susceptibility related to MIC creep? Open Access Maced J Med Sci 2018; 7:12-8.

Piewngam P and Otto M. Probiotics to prevent Staphylococcus aureus disease? Gut Microbes 2020; 11:94-101.

Raut S, Bajracharya K, Adhikari J, Pant S S and Adhikari B. Prevalence of methicillin resistant Staphylococcus aureus in Lumbini Medical College and Teaching Hospital, Palpa, Western Nepal. BMC Res Notes 2017; 10:187.

Sadeghi J and Mansouri S. Molecular characterization and antibiotic resistance of clinical isolates of methicillin-resistant Staphylococcus aureus obtained from Southeast of Iran (Kerman). APMIS 2014; 122:405-11.

Sahebnasagh R, Saderi H and Owlia P. The Prevalence of resistance to methicillin in Staphylococcus aureus strains isolated from patients by PCR method for detection of mecA and nuc Genes. Iran J Public Health 2014; 43:84-92.

Sakoulas G, Moellering R C Jr and Eliopoulos G M. Adaptation of methicillinresistant Staphylococcus aureus in the face of vancomycin therapy. Clin Infect Dis 2006; 42:40-50.

Satola S W, Farley M M, Anderson K F and Patel J B. Comparison of detection methods for heteroresistant vancomycin-intermediate

Staphylococcus aureus, with the population analysis profile method as the reference method. J Clin Microbiol 2011; 49:177-83.

Shariati A, Dadashi M, Moghadam M T, van Belkum A, Yaslianifard S and DarbanSarokhalil D. Global prevalence and distribution of vancomycin resistant, vancomycin intermediate and heterogeneously vancomycin intermediate Staphylococcus aureus clinical isolates: a systematic review and meta-analysis. Sci Rep 2020; 10: 12689.

Swenson J M, Anderson K F, Lonsway D R, Thompson A, McAllister $\mathrm{S} \mathrm{K}$, Limbago B M, Carey R B, Tenover F $\mathrm{C}$ and Patel $\mathrm{J}$ B. Accuracy of commercial and reference susceptibility testing methods for detecting vancomycin-intermediate Staphylococcus aureus. J clin microbiol 2009; 47:2013-7. 
Taha A E, Badr M F, El-Morsy F E and Hammad E. Prevalence and antimicrobial susceptibility of methicillin-resistant Staphylococcus aureus in an Egyptian University Hospital. J Pure Appl Microbiol 2019; 13:4.

Thati V, Shivannavar C T and Gaddad S M. Vancomycin resistance among methicillin resistant Staphylococcus aureus isolates from intensive care units of tertiary care hospitals in Hyderabad. Indian J Med Res 2011; 134:704-8.

Walsh T R, Bolmström A, Qwärnström A, Ho P, Wootton M, Howe RA, MacGowan AP and Diekema D. Evaluation of current methods for detection of Staphylococci with reduced susceptibility to glycopeptides. J clin microbiol 2001; 39:2439-44.

Wangai F K, Masika M M, Maritim M C and Seaton R A. Methicillin-resistant Staphylococcus aureus (MRSA) in East Africa: red alert or red herring? BMC Infect Dis 2019; 19:596.

Weissman J S, Adler A, Qin X and Zerr M D. Emergence of extended-spectrum $\beta$ lactam resistance among Escherichia coli at a US academic children's hospital is clonal at the sequence type level for CTX-M-15, but not for CMY2. Int J Antimicrob Agents 2013; 41: 414-20.

\section{How to cite this article:}

Maha Salah Elnady, Marwa Mohamed Ezzat, Azza Mahmoud Hassan, Amal Abd El Tawab Hasheesh and Mina Samy Messiha. 2021. Detection of Different Levels of Resistance to Vancomycin Among Staphylococcus aureus Isolated from Surgical Departments of Tanta University Hospitals. Int.J.Curr.Microbiol.App.Sci. 10(08): 402-413. doi: https://doi.org/10.20546/ijcmas.2021.1008.049 